\title{
The alternatively spliced domains EIIIB and EIIIA of human fibronectin affect
}

\section{cell adhesion and spreading}

\author{
Mariko Hashimoto-Uoshima1,*, Ying Zhuo Yan1, Galen Schneider ${ }^{2}$ and Ikramuddin Aukhil1,† \\ ${ }^{1}$ Department of Periodontics and Dental Research Center and ${ }^{2}$ Department of Prosthodontics and Dental Research Center, \\ University of North Carolina, Chapel Hill, NC 27599-7450, USA \\ *Present address: Tokyo Medical and Dental University, Tokyo, Japan \\ †Author for correspondence (e-mail: aukhil.drc@mhs.unc.edu)
}

\section{SUMMARY}

Fibronectin has a complex pattern of alternative splicing at the pre-mRNA level leading to the expression of different isoforms. The alternatively spliced domains EIIIB and EIIIA are known to be prominently expressed during development and wound healing. While the other spliced domain (CS-segment) is known to promote cell adhesion in a cell type specific manner, the biological functions of the spliced domains EIIIB and EIIIA are not well understood. In the present study, we have prepared expression proteins of specific domains of human fibronectin using a prokaryotic expression system and used the purified fragments to test their ability to support adhesion and spreading of cultured cells. Fragments from type-III domains \#7 to \#12 were prepared in various combinations to include or exclude the spliced domains EIIIB and EIIIA. The results indicate that cultured NIL fibroblasts adhere to many of the fragments tested. However, the cell adhesion and spreading are enhanced, especially at lower concentrations,

\section{INTRODUCTION}

The biological role of the extracellular matrix glycoprotein fibronectin (FN) in cell adhesion and migration, development, and wound healing has been extensively studied (Hynes, 1990). Fibronectins (FNs) are a group of related glycoproteins that arise from alternative splicing of the single FN gene transcript. FNs have a relatively simple structure and consist of a dimer of two subunits joined by disulfide bonds near the $\mathrm{COOH}$ termini (Hynes, 1990). Three types of homologous repeating units (referred to as types I, II and III domains/repeats) form each subunit. Alternative splicing of a single primary transcript leads to the inclusion or exclusion of three domains in the central part of the molecule. Among the various functions of FN, cell adhesion is by far the most extensively studied. The short polypeptide sequence Arg-Gly-Asp (RGD), located in the 10th type-III repeating unit of FN, has been identified as the important binding site for several integrin cell surface receptors. The other cell adhesion site identified within FN is referred to as the III CS (connecting segment) and this domain is also subject to alternative splicing at the pre-mRNA level (Humphries et al., 1987, 1988). The biological function(s) of to fragments including the domain EIIIB. The inclusion of domain EIIIA led to a decrease in the adhesion of cells and those that adhered did not spread well. When tested in a centrifugal cell adhesion assay, fragments including domain EIIIB resisted the detaching forces and stayed adhered. Fragments that included domain EIIIA were unable to resist the detaching centrifugal forces to the same extent as the fragments that included domain EIIIB alone. These results suggest that the spliced domain EIIIB may be serving important biological functions in enhancing cell adhesion and spreading. This is likely to be mediated by conformational effects because domain EIIIB alone neither exhibited any adhesive activity nor competed in inhibiting adhesion to fragments \#7-10.

Key words: Fibronectin, Domain EIIIB, Domain EIIIA, Cell adhesion, Extracellular matrix the two remaining alternatively spliced domains EIIIB and EIIIA is/are not known. It has, however, been well documented that the domains EIIIB and EIIIA of FN are prominently expressed during embryonic development and this embryonic pattern of mRNA expression reappears in healing wounds (ffrench-Constant and Hynes, 1989; ffrench-Constant et al., 1989). The expression of FN mRNA, including domains EIIIB and EIIIA, in the early chick embryo coincides at stages where there is active proliferation and migration of cells (ffrenchConstant and Hynes, 1989). Findings from a more recent study suggest that the alternatively spliced domains EIIIB and EIIIA are included in $\mathrm{FN}$ required for morphogenesis of ' $\mathrm{FN}$ dependent' structures during development (Peters and Hynes, 1996). Using immunohistochemistry, it has also been shown recently that the spliced domain EIIIB is included in FN around the walls of some smaller blood vessels and within cartilaginous structures (Peters et al., 1996). The possibility exists that these spliced domains of FN (EIIIB and EIIIA) may have important biological functions in the regulation of critical cellular events during embryonic development and wound healing.

Many of the biological functions of FNs have been mapped 
using proteolytic fragments (reviewed by Hynes, 1990). These types of studies have several disadvantages including overlapping functional sites and the lack of information on the isoform in question. Potentially, there may be as many as 20 different isoforms of FNs possible due to alternative splicing of the primary transcript (Hynes, 1990). Hence, studies aimed at mapping the biological functions of the spliced domains EIIIB and EIIIA should focus on methods that will provide clean fragments that begin and end precisely at individual domains. In the present study, we have generated several types of recombinant polypeptide fragments of human FN by amplifying cDNA coding for the type-III domains \#7-12 and cloning them into prokaryotic expression vectors. By including or excluding the spliced domains EIIIB and EIIIA, we were able to obtain ten different fragments in various combinations of the domains. These expressed domains of human FN were then purified and used to study cell adhesion. In this study, we demonstrate that the inclusion of spliced domain EIIIB alone or in combination with EIIIA, within the larger fragment of FNIII \#7-12, has an enhancing effect on the adhesion and spreading of NIL fibroblasts. However, the spliced domain EIIIA alone, within the fragment FNIII \#7-12, led to a reduction in the adhesion and spreading of cells. Since the domain EIIIB did not reveal any cell adhesion promoting activity when tested as a singledomain fragment, it is concluded that the domain EIIIB may be influencing cell adhesion by exerting conformational effects on the molecule.

\section{MATERIALS AND METHODS}

\section{Materials}

Human plasma FN (pFN) was provided by Dr Harold Erickson, Duke University, Durham NC. The monoclonal antibody 16G3, which has its epitope in the 10th type-III domain of human FN, was provided by Drs K. Yamada and S. Aota, NIDR, NIH. The mAB 7F9 to vinculin was provided by Dr A. Belkin. The prokaryotic expression vectors pET $11 \mathrm{~b}$ and pET 15b were from Novagen, Inc, Madison, Wis., and the pGEM vector was from Promega Corp. Oligonucleotide primers were synthesized at the Pathology Department Oligonucleotide Facility at the University of North Carolina Chapel Hill using an ABIModel 394 Machine. [ ${ }^{35}$ S ]dATP was from Amersham Corp, Isopropyl $\beta$-D-thiogalactosidase (IPTG) was from Gibco, and all other enzymes/reagents were from New England Biolabs or Promega.

\section{Cloning specific domains of human FN into pET expression vectors}

The methods used to produce and purify bacterial expression proteins were essentially the same as described previously with some modifications (Aukhil et al., 1993). The prokaryotic expression vector $\mathrm{pET}$ $11 \mathrm{~b}$ (Studier et al., 1990) and its modified version pET $15 \mathrm{~b}$ with a histidine tag to facilitate affinity chromatography were used. The strategy we have used enables us to clone fragments that begin and end precisely at the boundaries of individual domains of FN. Briefly, oligonucleotide primers with the appropriate restriction sites were designed to amplify fragments of human FN cDNA. The cDNA template used was made by reverse transcription of total RNA extracted from cultured human periodontal ligament fibroblasts. The various recombinant human FN fragments that include or exclude the alternatively spliced domains EIIIB and EIIIA are shown in Fig. 1. Both the forward and reverse oligonucleotide primers for DNA amplification shown in Table 1 were synthesized (18-21 bases) corresponding to the $5^{\prime}$ and $3^{\prime}$ terminal sequences of the desired coding segment. The forward primer was designed to incorporate the NdeI site imme- diately before the first codon and included the ATG start codon in the last three bases of its restriction sequence. The reverse primer included a stop codon after the codon for the last amino acid in the fragment to be amplified and was the followed by a BamHI site to facilitate directional cloning into $\mathrm{pET}$ vectors. Total RNA was isolated from cultured human periodontal ligament fibroblasts in their 4th and 5th passages and reverse transcribed using random hexamers (Kawasaki, 1990). The reverse transcription product was used as a template to amplify domains of human FN using Taq polymerase as described (Aukhil et al., 1993). Amplification of cDNA was verified by running samples on agarose gels and the amplified product was restriction digested with the appropriate enzymes. The fragments were initially cloned into pGEM-7 vector and their sequence verified by the dideoxynucleotide method (Sanger et al., 1977). Subsequently, the cDNA fragments were cloned into the NdeI and BamHI sites of the vector pET $11 \mathrm{~b}$ or pET 15b. Fragments including FN Type-III domain \#9 were subjected to limited digestion with Bam $\mathrm{HI}$ because of an intrinsic restriction site in this domain. Initially, the fragments FN-III domains 7-10(-B), $7-10(+\mathrm{B}), 10-12(-\mathrm{A})$ and $10-12(+\mathrm{A})$ were made and sequenced. The larger fragments and their combinations were made by using restriction digested segments. For example, the fragment Fn-III 7-12+B+A was assembled by using the $P v u I I$ digested fragments of $7-10+\mathrm{B}$ and 10-12+A. The fragment coding for domains 7-12-B-A was assembled using the Eco-0109 site in domain \#10. The same method was used to make 7-12+B-A as 7-12-B-A using enzyme-digested fragments from $7-10+\mathrm{B}$ and $10-12-\mathrm{A}$. The fragment $7-12-\mathrm{B}+\mathrm{A}$ was made from 7-10-B and 10-12+A using Bam HI digested pET $11 \mathrm{~b}$ containing 7-10 fragment as the second generation vector.

\section{Protein expression and purification}

Escherichia coli strain BL21(DE3) was transformed with pET 11b or pET $15 \mathrm{~b}$ plasmid DNA with the various fragments of human FN cloned into them. FN7-10(-B), 7-10(+B) and 7-B were cloned into $\mathrm{pET} 15 \mathrm{~b}$ and all other fragments were cloned into pET 11b. Overnight bacterial cultures were pelleted and the washed pellet resuspended in fresh LB medium with ampicillin $(50 \mu \mathrm{g} / \mathrm{ml})$. After a 3 hour period of growth in 1 liter of LB with ampicillin, IPTG was added at a final concentration of $2 \mathrm{mM}$ and the cultures were shaken at $37^{\circ} \mathrm{C}$ for another 3 hours. The protocol for induction and purification of the expressed proteins has been described previously (Aukhil et al., 1993). All the expressed proteins were soluble (seen in the supernatant after cell lysis and centrifugation). Ammonium sulfate precipitation was the first step followed by ion exchange chromatography on a Mono-Q column (Pharmacia). The proteins eluted as single peaks at various salt concentrations ranging from $0.2-0.4 \mathrm{M} \mathrm{NaCl}$. The peak fractions were pooled and dialyzed thoroughly against $20 \mathrm{mM}$ Tris$\mathrm{HCl}, \mathrm{pH}$ 7.9. Analysis of the purified protein samples on SDS-PAGE revealed clean single bands. However, upon long term storage at $4^{\circ} \mathrm{C}$ (more than 4 weeks) some proteins showed minor bands suggesting limited proteolysis. The proteins cloned into $\mathrm{pET} 15 \mathrm{~b}$ were purified by affinity chromatography on HIS-BIND resin columns (Novagen) that were charged with $\mathrm{Ni}^{2+}$. The bound proteins were eluted using Imidazole, dialyzed against $20 \mathrm{mM}$ Tris- $\mathrm{HCl}, \mathrm{pH} 7.4$, were further purified on the Mono-Q column and the pure fractions finally dialyzed against $20 \mathrm{mM}$ Tris-HCl, $\mathrm{pH}$ 7.9. Protein concentrations were determined by measuring the absorbance at $278 \mathrm{~nm}$. The extinction coefficient for each protein was calculated and estimated as described previously (Aukhil et al., 1993). SDS-PAGE was carried out using 5-20\% acrylamide gradient gels under reducing conditions and stained with Coomassie Blue.

\section{Protein adsorption assay}

The binding of $\mathrm{pFN}$ and the recombinant $\mathrm{FN}$ polypeptides was analyzed using an ELISA assay. Briefly, wells in 96-well flat bottom plastic plates (Falcon) were coated with various concentrations of each polypeptide in PBS (in triplicate) and incubated overnight at $4^{\circ} \mathrm{C}$. The solution was removed and the wells were washed $3 \times$ in PBS. The 
unbound sites within the wells were blocked by incubating heatdenatured BSA $\left(2 \mathrm{mg} / \mathrm{ml}\right.$, denatured at $80^{\circ} \mathrm{C}$ for 10 minutes) for 1 hour. The wells were again washed $3 \times$ with PBS containing $0.5 \%$ Tween-20. The rat monoclonal antibody $16 \mathrm{G} 3$ was added $(10 \mu \mathrm{g} / \mathrm{ml}$, $100 \mu \mathrm{l} /$ well) to each well and incubated for 2 hours at room temperature. The wells were then washed thoroughly $3 \times$ in PBS/Tween and then incubated with a peroxidase-conjugated goat anti-rat IgG for 2 hours. Finally, the wells were washed again in PBS/Tween and peroxidase activity was measured using $o$-phenylenediamine as a substrate. The reaction was stopped with $3 \mathrm{M} \mathrm{H}_{2} \mathrm{SO}_{4}$. The mount of polypeptides adsorbed to the well was expressed as the absorbance at $492 \mathrm{~nm}$. Control wells where only heat-denatured BSA was added were used to determine background levels.

\section{Cell culture}

Nil.8M hamster fibroblasts were obtained from Dr Richard Hynes, MIT. Cells were cultured in DMEM-H (Dulbecco's modified Eagle's medium with high glucose) with $10 \%$ fetal bovine serum and antibiotics (penicillin, streptomycin and gentamycin). The evening before the cell adhesion assays, cells were detached with $0.05 \%$ trypsin, 0.53 mM EDTA and replated at about 50\% confluence. The next day the cells were detached again and the effects of trypsin stopped by adding $0.5 \mathrm{mg} / \mathrm{ml}$ soy bean trypsin inhibitor. The cells were washed twice in PBS and then resuspended in DMEM-H (with no serum) and kept on ice until use.

\section{Gravity cell attachment assay}

The assay was carried out as described by Aukhil et al. (1993) and Joshi et al. (1993). Wells of 96-well flat bottom polyvinylchloride plastic microtiter plates (Falcon 3912) were coated with $100 \mu \mathrm{l}$ of various concentrations of the recombinant proteins in PBS overnight at $4{ }^{\circ} \mathrm{C}$. The wells were then washed in PBS and the unbound sites in the wells were blocked by adding $1 \mathrm{mg} / \mathrm{ml}$ heat denatured bovine serum albumin (BSA, denatured by heating to $80^{\circ} \mathrm{C}$ for 10 minutes, then cooled to room temperature) for 1 hour at room temperature. After washing the wells three times in PBS, each well was loaded with $100 \mu$ DMEM containing $5-10 \times 10^{4}$ cells suspended in the medium. The plates were incubated for 60 minutes at $37^{\circ} \mathrm{C}$ in an atmosphere of $5 \% \mathrm{CO}_{2}$. Following the incubation period, the wells were washed $3 \times$ with PBS to remove the unbound cells. The bound cells were fixed and stained with $3.7 \%$ formaldehyde and $1 \%$ Toluidine Blue or $0.1 \%$ Crystal Violet in PBS for a minimum of 2 hours to overnight at $4^{\circ} \mathrm{C}$. After washing to remove the unbound dye, the bound cells were lysed with $2 \%$ SDS in PBS. The intensity of the color was measured at 595 $\mathrm{nm}$. A blank value corresponding to BSA-coated wells was subtracted from each value. Data from triplicate wells were averaged for each time point/protein concentration tested. The standard deviation of the three wells was typically within $10 \%$ of the mean. The assay was repeated at least three times to establish reproducibility.

To study the spreading and morphology of cells, $10 \mu \mathrm{l}$ samples of the proteins at a concentration of $50 \mathrm{nM}$ were spread on marked circular spots (6 $\mathrm{mm}$ in diameter) in $60 \mathrm{~mm}$ tissue culture dishes (Corning). After overnight incubation at $4{ }^{\circ} \mathrm{C}$, the unbound proteins were removed by aspirating the remaining solution from marked areas. The dishes were washed in PBS $3 \times$, unbound sites blocked with $\mathrm{BSA}$, and the cells plated. After 60 minutes of incubation at $37^{\circ} \mathrm{C}$, the dishes were washed and the bound cells fixed/stained as before. The adherent cells were examined using an inverted microscope and photographed.

The presence of focal adhesions and actin stress fibers in cells plated on the recombinant FN fragments were analyzed using immunofluorescence methods as described by Schneider and Burridge (1994). Briefly, the recombinant FN fragments or native plasma FN were coated on individual glass coverslips and allowed to bind for 16 hours. The coverslips were washed in PBS twice and the unbound sites blocked with heat denatured BSA for 60 minutes. NIL cells were trypsinized, washed, plated on these coverslips (approx. 2,000 cells), and then placed into 24 -well plates. After a 60 minute incubation at $37^{\circ} \mathrm{C}$, the medium was removed and the wells with the glass coverslips were washed gently three times in PBS. The bound cells were fixed in $3.7 \%$ formaldehyde for 10 minutes, then washed in Trisbuffered saline (TBS: $50 \mathrm{mM}$ Tris- $\mathrm{HCl}, 150 \mathrm{mM} \mathrm{NaCl}, 0.1 \% \mathrm{NaN} 3$, $\mathrm{pH}$ 7.6) for 5 minutes. The coverslips, with the cells attached to them, were incubated sequentially in $0.5 \%$ Triton for 7 minutes, TBS for 5 minutes, primary antibody to vinculin (mAB 7F9) for 60 minutes at $37^{\circ} \mathrm{C}$, TBS for 5 minutes, secondary antibody (DAM-R, Chemicon, 1:25 in TBS), TBS for 5 minutes, dipped into water and then mounted on Mowiol (Calbiochem. Corp.). The coverslips were examined under a Zeiss fluorescence microscope and the images captured on T-MAX film (Kodak). To identify actin, coverslips were stained with phalloidin (Molecular Probes) at 1:500 dilution.

\section{Centrifugal cell adhesion assay}

96-well U-bottom polyvinylchloride plastic microtitre plates (Falcon 3911) were coated with $100 \mu \mathrm{l} /$ well of the different concentrations of the recombinant proteins in PBS by overnight adsorption at $4^{\circ} \mathrm{C}$. The wells were washed and unbound sites blocked with $10 \mathrm{mg} / \mathrm{ml}$ of BSA in PBS. $100 \mu \mathrm{l}$ of a cell suspension containing $5-10 \times 10^{3}$ cells were placed in each well and the cells allowed to settle/adhere for 15 minutes at $37^{\circ} \mathrm{C}$ in an atmosphere of $5 \% \mathrm{CO}_{2}$. Following this incubation period, the plate was centrifuged at $250 \mathrm{~g}$ for 5 minutes at $4{ }^{\circ} \mathrm{C}$ in a Sorval RT6000B centrifuge. The medium in the wells was gently removed and replaced with $17.5 \%$ formaldehyde with $0.005 \%$ Crystal Violet in PBS for fixation for at least one hour. The fixative was then removed and the wells washed gently in PBS. The effects of centrifugation on the adhesion of cells to the coated ligands was determined by examining the cells in the round bottom wells and scoring as follows. Wells that showed complete pelleting of the cells were scored as 0 (e.g. BSA coated wells), wells that displayed maximum resistance to centrifugation and showed adherent cells received a score of 3 (e.g. plasma fibronectin), wells with cells showing more adherent cells than pelleted cells received a score of 2 , and finally cells that showed more pelleted cells than adherent cells received a score of 1 . In addition, the center of each well was photographed.

\section{RESULTS}

\section{Biochemical characterization of the bacterial expression proteins}

A total of ten different expression proteins corresponding to the various type-III domains of human FN were made and their nomenclature is shown in Fig. 1. The oligonucleotide primers used to amplify the domain specific recombinant fragments of human FN are shown in Table 1. All the cDNA fragments were sequenced to verify the fidelity of DNA amplification by Taq polymerase. When cloned into the prokaryotic expression vector $\mathrm{pET} 11 \mathrm{~b}$ and induced by IPTG, all of them expressed the protein well with the yield varying between constructs. All the recombinant proteins were soluble and could be easily purified by a combination of ammonium sulfate precipitation followed by ion exchange chromatography on Mono-Q columns. When necessary, an additional step of gel filtration was carried out on Superdex-75 column. The purified expression proteins were analyzed by SDS-PAGE, and demonstrated high levels of purity (Fig. 2). Storage at $4^{\circ} \mathrm{C}(>4$ weeks) in some instances led to a partial degradation, as evidenced by doublet bands that stained with the monoclonal antibody to domain \#10 on western blots (data not shown). Hence experiments were carried out using fresh batches (within 2-3 weeks afer purification) of recombinant $\mathrm{FN}$ fragments. The molecular masses 


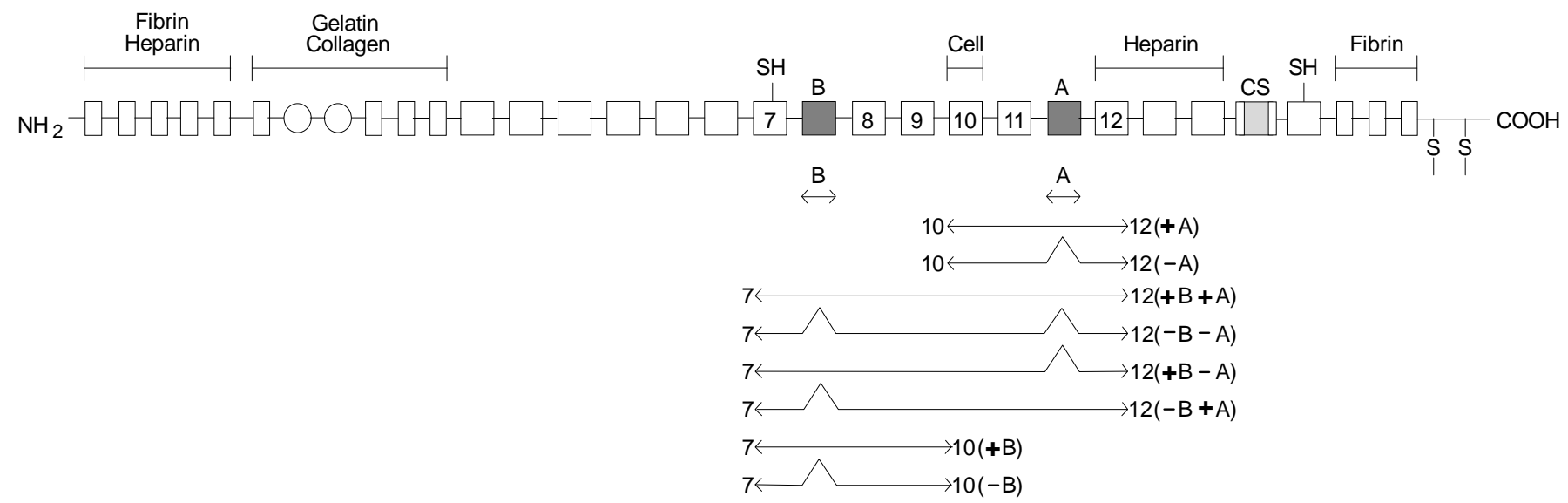

Fig. 1. Diagrammatic illustration of the recombinant human FN fragment constructs. The primers for amplification of the cDNA were designed to begin and end precisely at the various domain boundaries.

and extinction coefficients for each of these recombinant fragments are shown in Table 2. Fragments made in pET 15b with the histidine tag showed no difference in cell adhesion assays compared to similar fragments made in pET $11 \mathrm{~b}$ without the histidine tag.

\section{Adsorption of recombinant FN fragments to plastic surface}

A modified ELISA was used to determine the binding of FN fragments to wells of plastic plates used for cell adhesion assays. Using the monoclonal antibody 16G3 to the 10th typeIII repeat of FN (Nagai et al., 1994), the four large fragments (FN \#7-12 with and without IIIB and IIIA) and the four smaller fragments (FN \#7-10+/-IIIB; FN \#10-12+/-IIIA) were tested for adhesion to plastic at concentrations ranging from 1 to 100 nM. The data are shown in Fig. 3 and all eight recombinant fragments showed adsorption to plastic wells. Since the monoclonal antibody was used at only one concentration, it was found to be insufficient giving a premature saturation reading for the binding of protein fragments. The apparent saturation of the protein fragments at $50 \mathrm{nM}$ concentration was later found to be inaccurate since cell adhesion assays showed increasing adhesion for some fragments up to $100 \mathrm{nM}$ concentration.

\section{Cell adhesion by gravity assays and effects on cell spreading}

The results from gravity cell attachment assays are shown in Fig. 4. Human plasma FN was used as positive control, and as expected, showed good adhesion at $50 \mathrm{nM}$ concentration. The construct FN \#7-10+B also showed adhesion at $50 \mathrm{nM}$ that was comparable to plasma FN. The absence of spliced domain IIIB from the FN \#7-10 construct reduced the adhesion by approximately $50 \%$, and even at concentrations of $200 \mathrm{nM}$ had not reached that seen for FN \#7-10+B at $50 \mathrm{nM}$ (as per assays in multiwell plates). The constructs FN \#10-12 with and without IIIA showed significantly less adhesion of cells even at concentrations as high as $200 \mathrm{nM}$. In general, the presence of adjacent domains at the N-terminal end of the 10th type-III domain with the RGD sequence appeared to favor cell adhesion and this was enhanced when domain EIIIB was included. The signifance of domains at the $\mathrm{N}$-terminal end of the tenth domain
(FN III \#7,8,9) in enhancing adhesion was further confirmed by testing cell adhesion to larger fragments that contained type-III domains both at the N-terminal (FNIII \# 7-9) and C-terminal ends (FNIII \#11,12) of the tenth type-III domain. For example, as shown in Fig. 4, the order of cell adhesion to these fragments was $(\# 7-12+\mathrm{B}-\mathrm{A})>(\# 7-12+\mathrm{B}+\mathrm{A})>(\# 7-12-\mathrm{B}-\mathrm{A})>(\# 7-12-$ $\mathrm{B}+\mathrm{A})$. In both the smaller as well as the larger fragments, the inclusion of domain IIIA appeared to reduce the adhesion of cells while the inclusion of IIIB appeared to enhance it.

\section{Immunofluorescence staining for focal adhesions and cytoskeleton}

NIL cells plated on glass coverslips coated with the various recombinant $\mathrm{FN}$ fragments were stained with $\mathrm{mAB}$ to vinculin

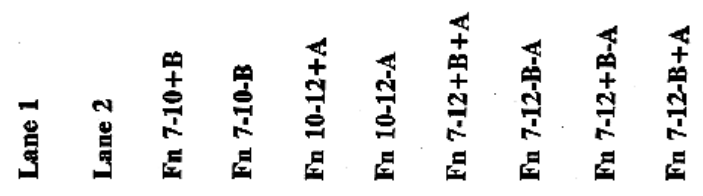

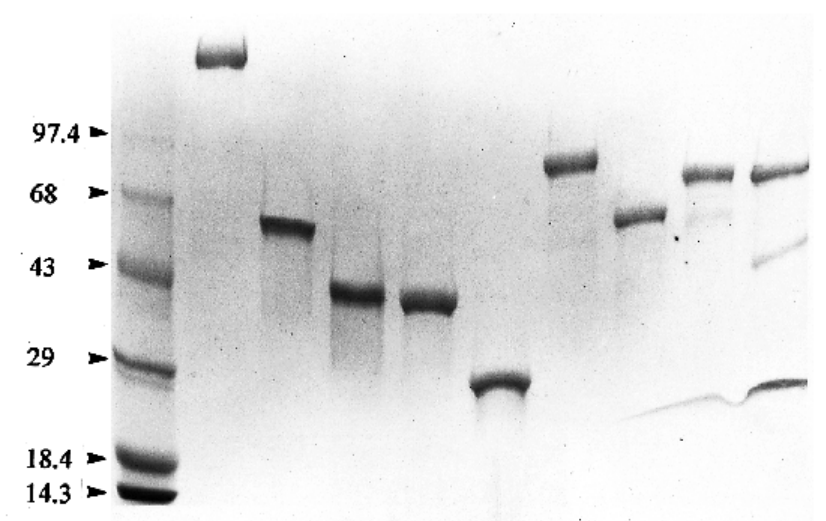

Fig. 2. SDS-PAGE of purified recombinant human FN fragments. Samples were run on a 5-20\% acrylamide gel and stained with Coomasie blue. Lane 1 is molecular mass markers $(\mathrm{kDa})$, lane 2 is plasma FN and the purified recombinant human FN fragments, all purified from the $\mathrm{pET}$ expression vector are shown as marked above the lanes. 
Table 1. Oligonucleotide primers used to clone the fragments of human FN

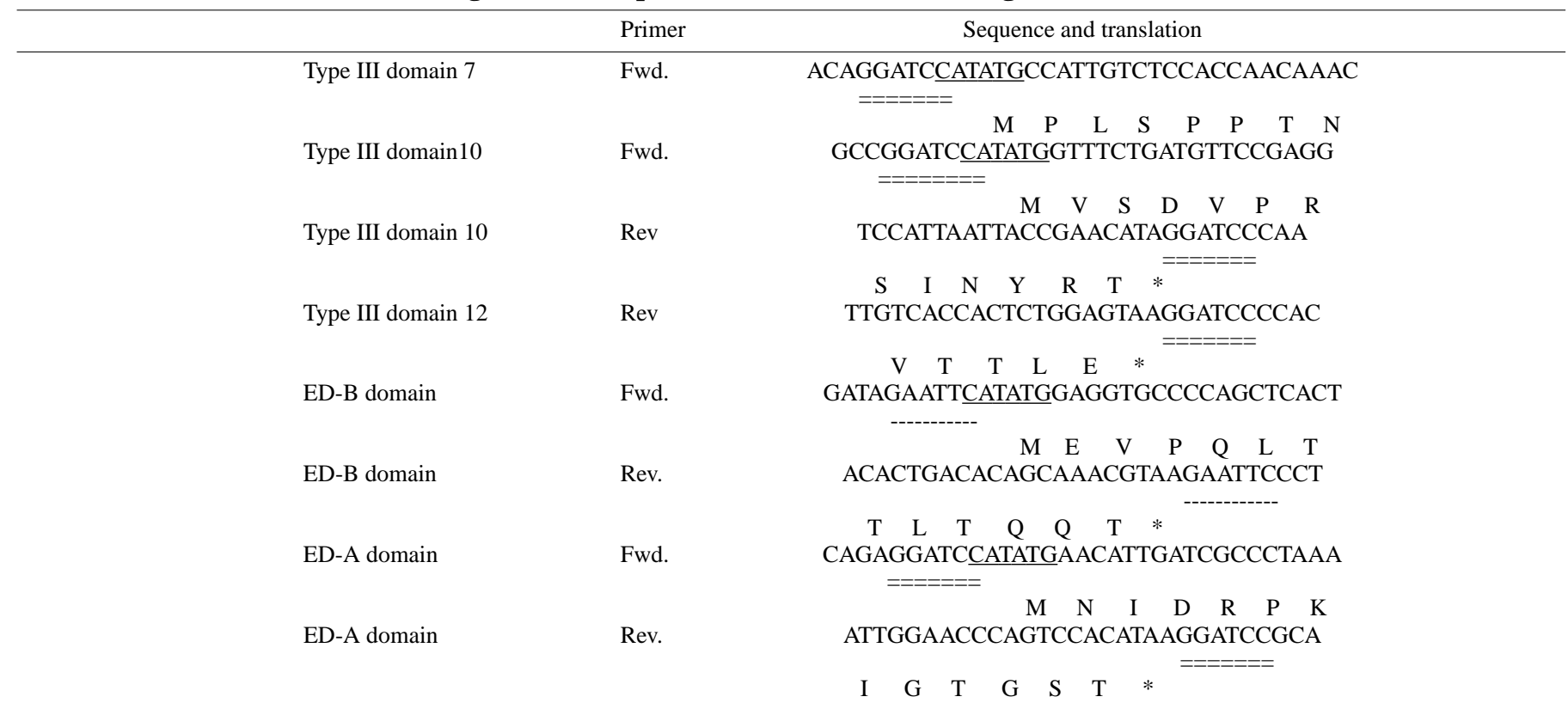

The reverse primer is listed as the sense strand (with translation), but the anti-strand was the sequence actually used. NdeI restriction sites are indicated by underline; BamHI sites are double underlined; EcoRI sites are indicated by -----. The asterisks show the stop codons.

to examine the focal adhesions and with phalloidin to examine the actin network. Since the gravity adhesion assays had shown differences between the abilities of the various fragments to allow adhesion of cells at $50 \mathrm{nM}$ concentrations, the coverslips were also coated at the same concentration. As shown in Fig. 5.1, the fragment FN\#7-10+IIIB showed cell spreading and strong staining for focal adhesions that was comparable to native plasma FN (Fig. 5.9). The focal adhesions were also more prominent in cells plated on FN\#7-10+IIIB (Fig. 5.1) than in cells plated on FN\#7-10-IIIB (Fig. 5.2). The fragments FN\#10-12-IIIA (Fig. 5.3) and FN\#10-12+IIIA (Fig. 5.4) showed very few cells adhering and virtually no spreading. No significant focal adhesions were detectable in cells plated on these fragments. In the larger FN\#7-12 fragments, the differences in focal adhesions were subtle, although the fragments FN\#7-12+IIIB+IIIA (Fig. 5.5), FN\#7-12-IIIB-IIIA (Fig. 5.6) and FN\#7-12+IIIB-IIIA (Fig. 5.7) showed slightly more spreading of cells and focal adhesions compared to the large fragment FN\#7-12-IIIB+IIIA (Fig. 5.8).
The organization of actin stress fibers was also examined in these cells by staining with phalloidin. Although the immunofluorescence was not as bright as that seen with staining for focal adhesions, the findings appeared to parallel those observed for focal adhesions and in the case of the larger fragments, the fragment FN7-12-IIIB+IIIA showed a less intense staining for actin as compared to the other fragments (Fig. 6).

\section{Centrifugal cell adhesion assay}

This assay was designed as an indicator of the relative strengths of cell adhesion to the various recombinant FN fragments. Examples of the scoring criteria (0-3) are shown in Fig. 7. Cells plated on native plasma $\mathrm{FN}$ showed resistance to centrifugal detaching forces fifteen minutes after plating them. Other proteins that offered resistance to detaching centrifugal forces at low concentrations (50 $\mathrm{nM})$ were FN\#7-10+IIIB and FN\#710-IIIB. The FN\#10-12 fragments showed almost complete pelleting at $50 \mathrm{nM}$ concentrations. In comparison, the larger

Table 2. Biochemical characterization of recombinant fragments of human FN

\begin{tabular}{|c|c|c|c|c|c|c|}
\hline \multirow[b]{2}{*}{ Peptide } & \multicolumn{3}{|c|}{ Molecular mass } & \multicolumn{3}{|c|}{$\varepsilon$} \\
\hline & Native protein & $\mathrm{pET} 11 \mathrm{~b}$ & $\mathrm{pET} 15 \mathrm{~b}$ & Native protein & $\mathrm{pET} 11 \mathrm{~b}$ & $\mathrm{pET} 15 \mathrm{~b}$ \\
\hline PFn & 230,000 & & & 1.28 & & \\
\hline $7-10+B$ & & $49,178.35$ & $51,354.54$ & & 1.082 & 1.036 \\
\hline $7-10-B$ & & $39,482.63$ & $41,658.82$ & & 1.064 & 1.008 \\
\hline $10-12+\mathrm{A}$ & & $38,094.32$ & $40,270.51$ & & 1.139 & 1.07 \\
\hline $10-12-\mathrm{A}$ & & $28,606.04$ & $30,782.23$ & & 1.175 & 1.09 \\
\hline $7-12+B+A$ & & $77,273.50$ & $79,449.69$ & & 1.069 & 1.03 \\
\hline $7-12-B-A$ & & $58,089.50$ & $60,265.69$ & & 1.060 & 1.02 \\
\hline $7-12+B-A$ & & $67,785.22$ & $69,961.41$ & & 1.074 & 1.04 \\
\hline $7-12-B+A$ & & $67,577.78$ & $69,753.97$ & & 1.057 & 1.02 \\
\hline
\end{tabular}

$\varepsilon=(\mathrm{Y} \times 1,400+\mathrm{W} \times 5,600)$ molecular mass. Y, Tyr; W, Trp.

$\mathrm{OD}_{278} / \varepsilon=$ protein concentration $(\mathrm{mg} / \mathrm{ml})$. 

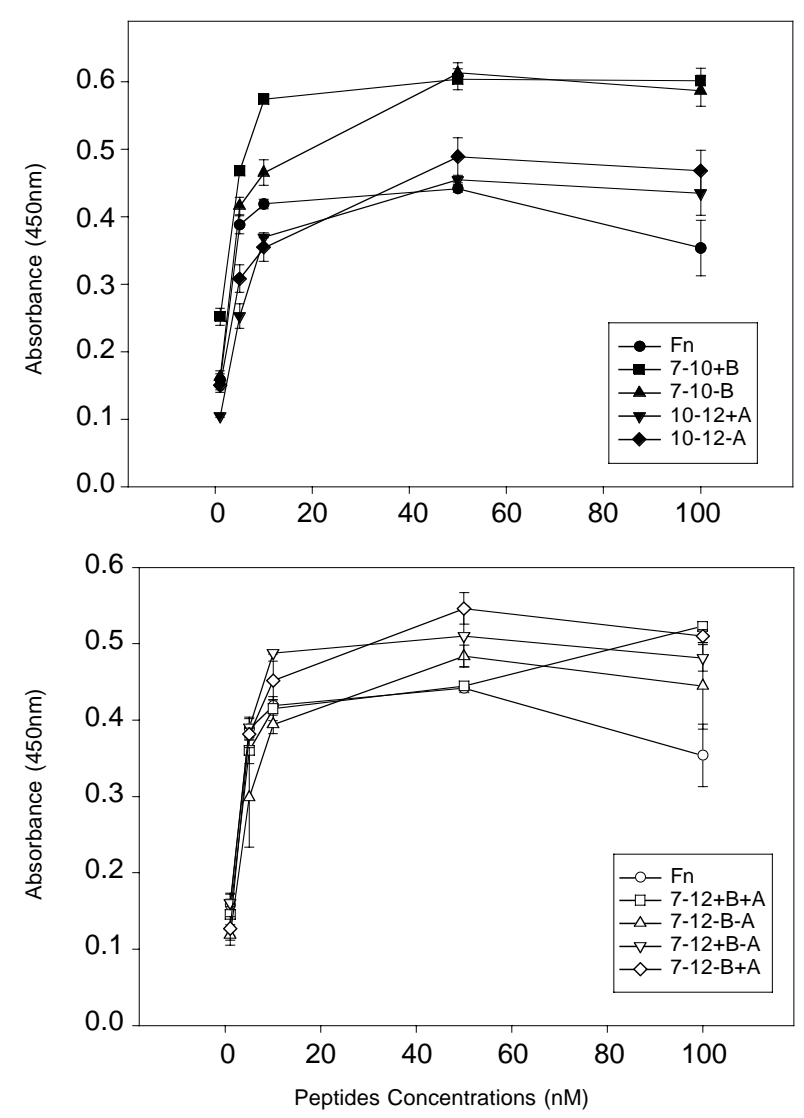

Fig. 3. Adsorption of recombinant human FN fragments to plastic surfaces. Wells in 96-well plates were coated with dilutions (in triplicate) of the various recombinant fragments overnight at $4{ }^{\circ} \mathrm{C}$. The next day, wells were washed three times with PBS and incubated with mAB 16G3 to human FN domain \#10 for 2 hours at room temperature. The wells were again washed three times with PBSTween and then incubated with HRP-conjugated secondary antibody for 2 hours. Finally, the wells were rinsed thoroughly with PBS before adding the substrate. Absorbance was read at $492 \mathrm{~nm}$ and the results shown are the means of triplicate values.

FN\#7-12 fragments showed mostly resistance to centrifugal detaching forces with the FN\#7-12+IIIB-IIIA showing the best results followed by the FN\#7-12+IIIB+IIIA fragment. These results were reproducible in three separate experiments using these recombinant fragments.

\section{DISCUSSION}

We have used recombinant fragments of type-III repeats of human fibronectin in the present study to examine the biological functions of the alternatively spliced domains EIIIB and EIIIA. The prokaryotic expression vectors pET15b and pET11b (with and without histidine tags respectively, for purification) yield recombinant fragments that possess biological activity in the form of promoting cell adhesion. The crystal structure of one four-domain fragment of human fibronectin fragment (FNIII 7-10), refined to $2.0 \AA$, prepared as described here, has been recently solved (Leahy et al., 1996), suggesting that the recombinant fragments fold properly. We encountered
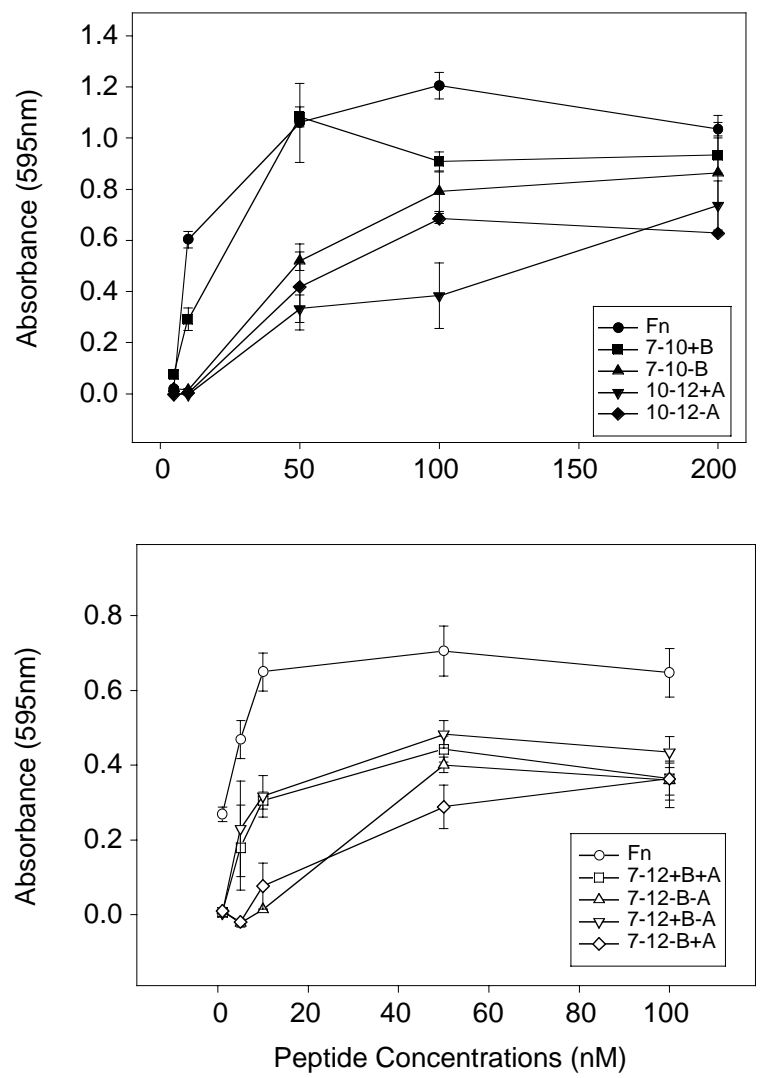

Fig. 4. Gravity cell attachment assay for quantification of cell adhesion to recombinant human FN fragments. Wells in 96-well plates were coated overnight with the various dilutions of the respective fragments and the unbound sites blocked with heat-denatured BSA. Cells were then plated and after 1 hour at $37^{\circ} \mathrm{C}$ wells were washed gently to remove unbound cells. Adherent cells were fixed and stained overnight in $3.7 \%$ formaldehyde with $0.1 \%$ Crystal Violet or $1 \%$ Toluedene Blue. Wells were again washed gently and the bound dye released by lysing the cells in $100 \mathrm{ml}$ of $2 \%$ SDS. The absorbance was read at 595 $\mathrm{nm}$. The data represent the mean of triplicate wells and the data trends were reproducible in three such experiments.

no technical difficulties in the production and purification of the fibronectin fragments with all the fragments being soluble in the bacterial lysate and easily purified by a combination of gel filtration, affinity (for hist-tag proteins made in pET 15b) and ion-exchange chromatography. The cloning of fragments based on amplification of cDNA to begin and terminate each protein at the boundaries of the independently folding domains successfully yields stable fragments of the FN-III domains. We had successfully used a similar approach to map the cell- and heparin-binding domains of human tenascin (Aukhil et al., 1993). In the present study, by using different combinations of type-III fragments 7-12 of human FN that include or exclude the spliced repeats EIIIB and EIIIA, we show that including the EIIIB repeat enhances the adhesion and spreading of cells. On the other hand including repeat EIIIA alone reduced the adhesion and spreading of cells, while including it along with EIIIB made no difference in terms of cell adhesion and spreading.

Recombinant fragments of human FN were made to include, in addition to the type-III repeat \#10 with the putative RGD cell 
binding loop, adjacent domains on both sides. Previous studies have shown that the presence of adjacent type-III domains \#9 and \#8 are needed to restore the ability of the putative RGDcontaining domain \#10 to support cell adhesion and spreading (Kimizuka et al., 1991). This along with the location of domains EIIIB (between 7 and 8) and EIIIA (between 11 and 12) dictated the design of our fragments that extended from repeats 7-12. The finding that inclusion of domain EIIIB alone in fragments FN 7-10 and 7-12 enhances the adhesion and spreading of cells is interesting and points to several possibilities. The first possibility is that the EIIIB domain itself has cell-adhesive properties via a different integrin, or uses the same alpha5 beta1 integrin. This was not found to be the case because domain EIIIB alone or fragment 7+EIIIB did not promote cell adhesion and were not successful in inhibiting adhesion to the 7-10+B fragment in competitive assays. Domain EIIIB alone showed no adhesion at concentrations of up to $10 \mu \mathrm{M}$. In competitive inhibition experiments (data not shown), trypsinized cells incubated with $50 \mu \mathrm{M}$ of purified fragment EIIIB in DMEM for 15 minutes with gentle shaking at $37^{\circ} \mathrm{C}$ before plating in wells coated with $50 \mathrm{nM}$ of the fragments $(\mathrm{FN}, 7-10,7-10+\mathrm{B})$ showed no inhibition of cell adhesion. The second possibility is that including domain EIIIB would generate a conformational modification in the domains downstream, perhaps improving the
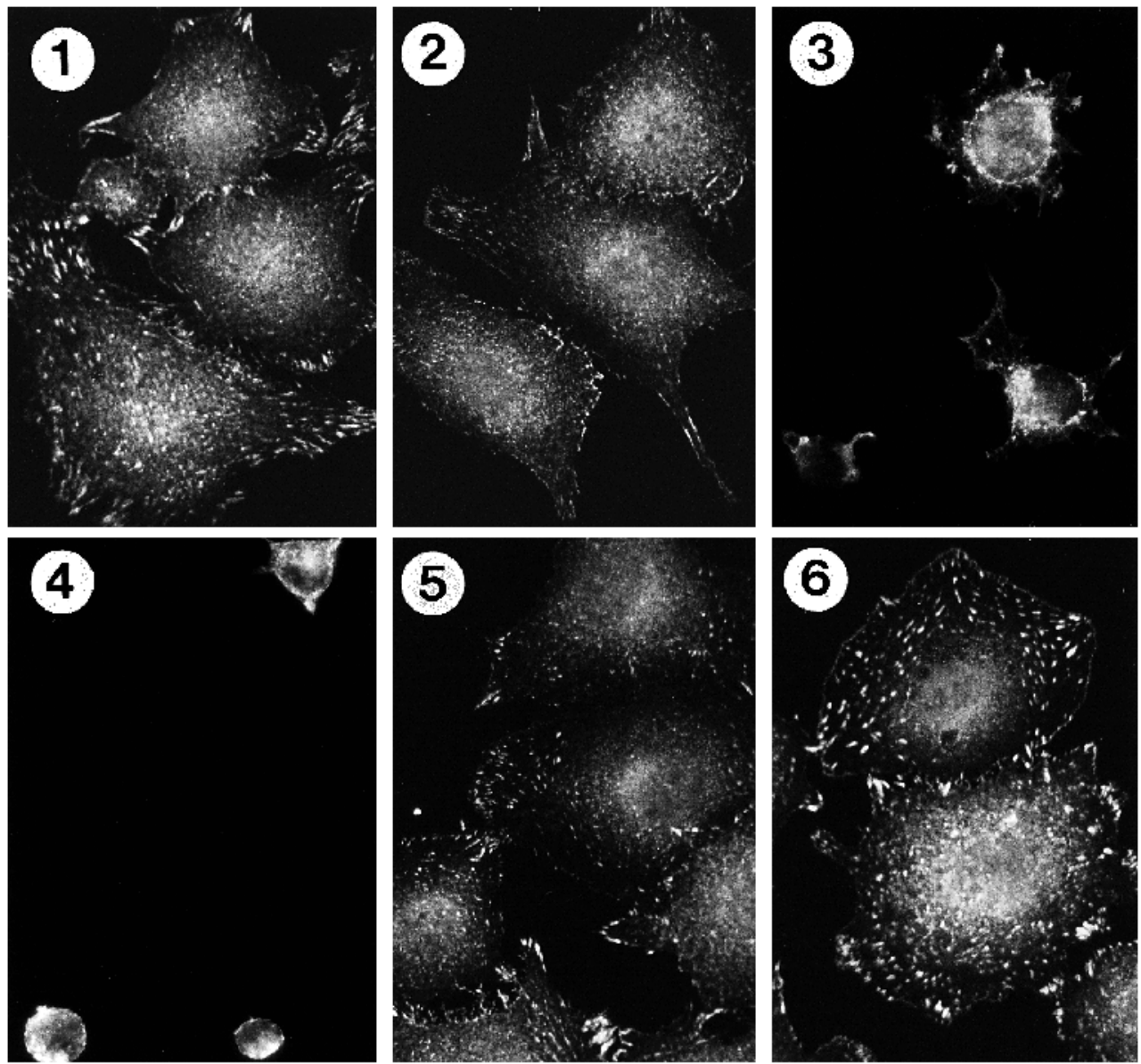

Fig. 5. Distribution of focal adhesions in cells plated on the various recombinant human $\mathrm{FN}$ fragments. Glass coverslips were coated with the proteins at $50 \mathrm{nM}$ concentration and unbound sites blocked with BSA. Cells were plated for 1 hour following which the glass coverslips were rinsed in PBS and processed for immunofluorescence staining of focal adhesions using antibody to vinculin. The coverslips were coated as follows : $1=7-10+\mathrm{B}$; $2=7-10-\mathrm{B} ; 3=10-12+\mathrm{A} ; 4=10-12-\mathrm{A}$; $5=7-12+\mathrm{B}+\mathrm{A} ; 6=7-12-\mathrm{A}-\mathrm{B} ; 7=7-$ $12+\mathrm{B}-\mathrm{A} ; 8=7-12-\mathrm{B}+\mathrm{A} ; 9=\mathrm{pFN}$.
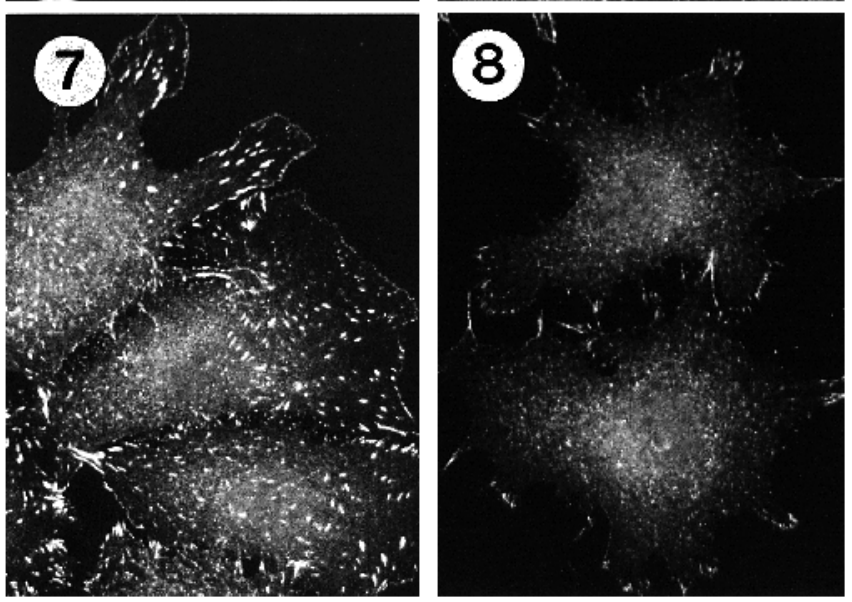
Table 3. Data from centrifugal cell attachment assay

\begin{tabular}{|c|c|c|c|c|c|c|c|c|c|}
\hline & $\mathrm{FN}$ & $7-10+B$ & $7-10-B$ & $10-12+\mathrm{A}$ & $10-12-\mathrm{A}$ & $7-12+B+A$ & $7-12-\mathrm{B}-\mathrm{A}$ & $7-12+B-A$ & $7-12-B+A$ \\
\hline $1 \mathrm{nM}$ & 0.5 & 0 & 0 & 0 & 0 & 0.5 & 0 & 0.5 & 0 \\
\hline $5 \mathrm{nM}$ & 1.5 & 2 & 0.5 & 0 & 0 & 1 & 0.5 & 1 & 0.5 \\
\hline $10 \mathrm{nM}$ & 3 & 2 & 1 & 0 & 0.5 & 1.5 & 1 & 2 & 0.5 \\
\hline $50 \mathrm{nM}$ & 3 & 3 & 3 & 0.5 & 1 & 2 & 2 & 2.5 & 1.5 \\
\hline $100 \mathrm{nM}$ & 3 & 3 & 3 & 1 & 1 & 2.5 & 3 & 3 & 1.5 \\
\hline $200 \mathrm{nM}$ & 3 & 3 & 3 & 1 & 1 & 3 & 2.5 & 3 & 2 \\
\hline $400 \mathrm{nM}$ & 3 & 3 & 3 & 1.5 & 1 & 3 & 2.5 & 3 & 2.5 \\
\hline $1,000 \mathrm{nM}$ & 3 & N.D. & N.D. & 2 & 2 & N.D. & N.D. & N.D. & N.D. \\
\hline
\end{tabular}

N.D., not determined.

Fig. 6. Distribution of actin stress fibers in cells plated on the various

recombinant human FN fragments. Glass coverslips were coated with the proteins at $50 \mathrm{nM}$ concentration and unbound sites blocked with BSA. Cells were plated for 1 hour following which the glass coverslips were fixed, subsequently rinsed and the actin stress fibers stained with phalloidin. The coverslips were coated as follows : $1=7-10+\mathrm{B} ; 2=7-10$ $\mathrm{B} ; 3=10-12+\mathrm{A} ; 4=10-12-\mathrm{A} ; 5=7$ -

$12+\mathrm{B}+\mathrm{A} ; 6=7-12-\mathrm{A}-\mathrm{B} ; 7=7-12+\mathrm{B}-\mathrm{A}$; $8=7-12-\mathrm{B}+\mathrm{A} ; 9=\mathrm{pFN}$.
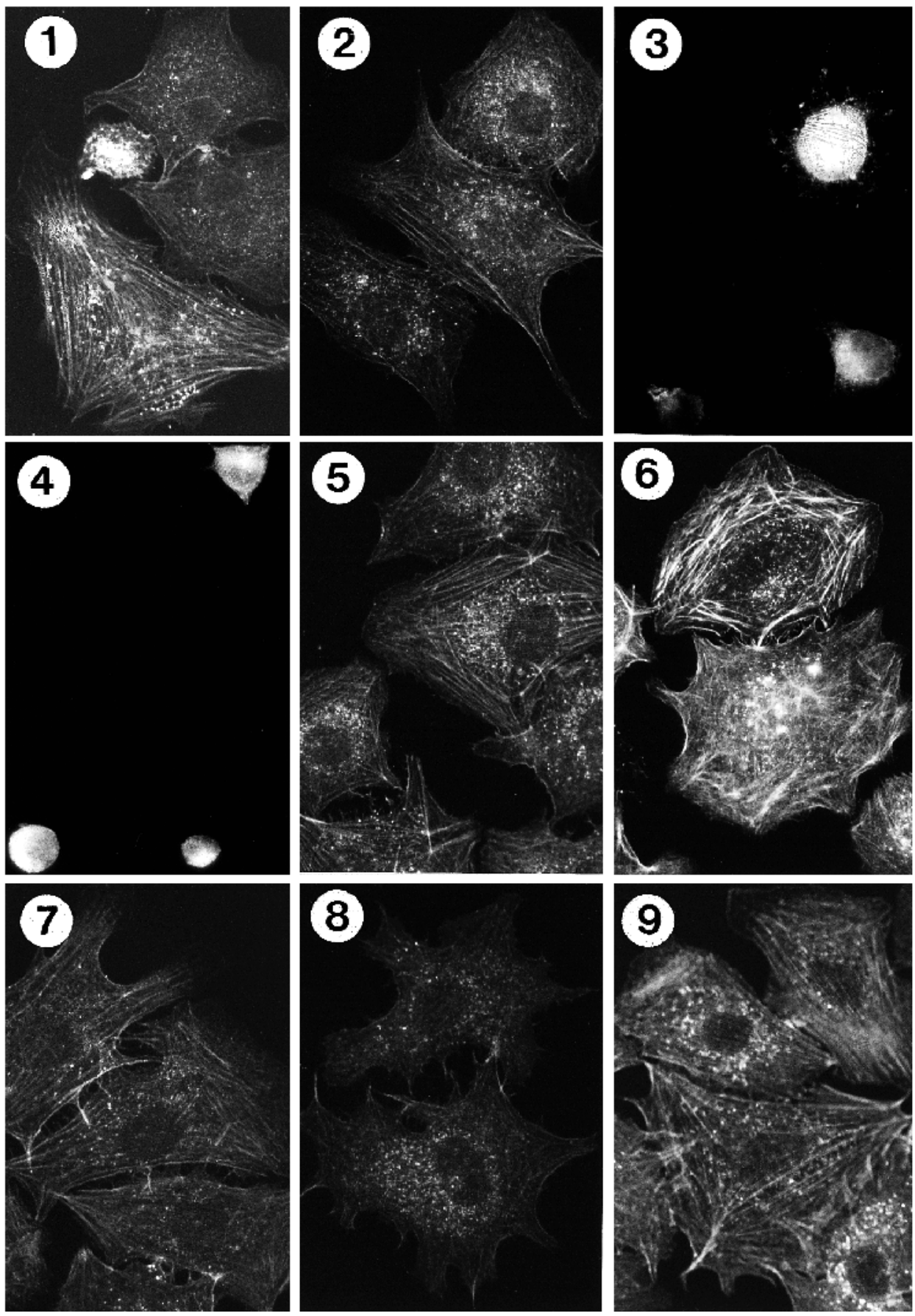
A

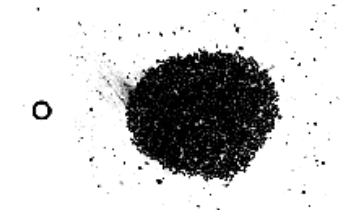

1
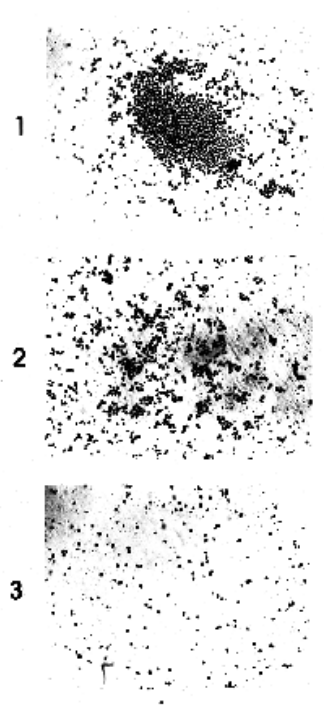

B
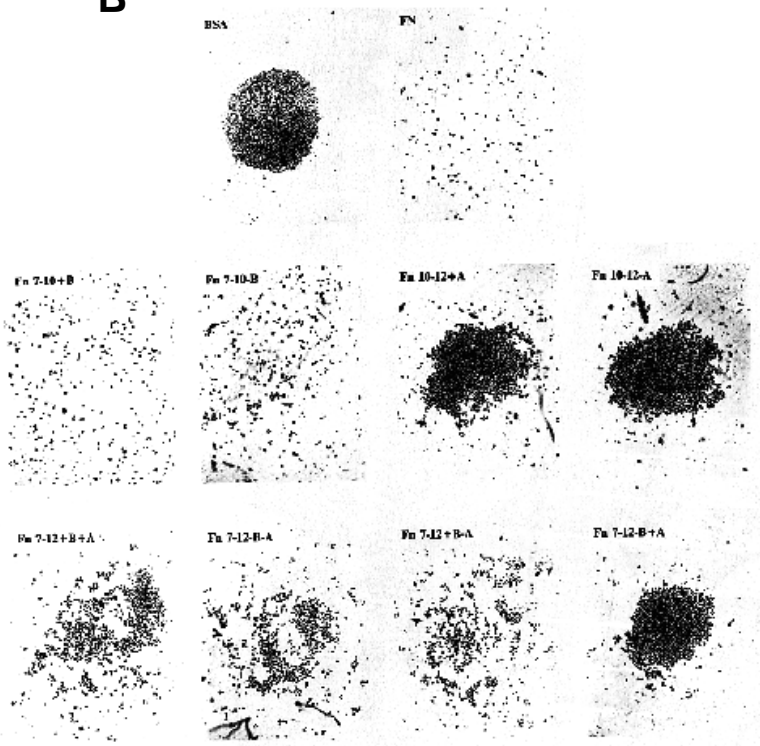

Fig. 7. Centrifugal cell adhesion assay. Round-bottom wells in 96well plates were coated with recombinant fragments of human FN overnight. After blocking the unbound sites with heat-denatured BSA, cells were plated and allowed to adhere for 15 minutes. The entire 96 plate was then centrifuged at $250 \mathrm{~g}$ for 5 minutes. The medium was then gently aspirated and the cells fixed with $3.7 \%$

formaldehyde $/ 0.1 \%$ Crystal Violet overnight. The wells were rinsed again and the center of the round-bottom wells examined and photographed. (A) Examples of scoring criteria are 0 for complete pelleting, 1 for mostly pelleting in the center with a few cells resisting detachment around the periphery, 2 for cells that are mostly resisting detachment but a few clusters at the center and, 3 for cells that have resisted detachment with no pelleting or clusters either in the center or in the periphery. (B) Examples of centrifugal assays on the various recombinant fragments of human FN. BSA is the negative control giving 0 scores and $\mathrm{pFN}$ is the positive control giving scores of 3 . access to integrin binding sites in domain 10 and 9. To this effect, a previous study by Carnemolla et al. (1992) had found that including the EIIIB repeat in their $\beta$-galactose-fibronectin fusion protein had unmasked cryptic sequences suggesting conformational effects of including EIIIB. More recently, the crystal structure of the recombinant human FN fragment 7-10 (Leahy et al., 1996) suggests some interesting structural consequences. The fragment FN 7-10, made up of four contiguous type-III modules shows relative rotations ranging from 43 to $160^{\circ}$. In addition, the solvent-accessible surface area buried between domains is as follows: $587 \AA$ between domains 7 and 8, $527 \AA$ between domains 8 and 9, and only $333 \AA$ between domains 9 and 10. It is also interesting to note that an almost twofold rotation axis relates domain 8 and 9. In summary, the small rotation and tilt angles relating domains 9 and 10 renders this segment of the 7-10 fragment almost cylindrical and contrasts with the combined tilts and rotations relating domains 7-9 giving that segment a spiral configuration (Leahy et al., 1996). While definite information on the structural effects of including EIIIB would require crystallographic analysis, it is tempting to speculate on the structural aspects. It is possible that the large interface between domains 7 and 8 contains sequences that may have synergistic functions in promoting the adhesion and spreading of cells. While these interface sequences are buried and may be masked in the [-EIIIB] isoform, the inclusion of EIIIB may be exposing these proximal sequences. The other possibility is that the inclusion of EIIIB may affect the polarity of the domains downstream resulting in the positioning of the RGD cell-binding site in domain 10 and the other recently-discovered synergy sequences (PHSRN) in domain 9 (Aota et al., 1994) to enhance the adhesion and spreading of cells. Since domain 9 contains more basic amino acids and is located immediately before domain 10, an extra domain that may influence the proper folding and conformation of the positively charged region in domain 9 and the RGDS site could be enhancing the biological function of the synergy sequences (Kimizuka et al., 1991). The final possibility is that the NIL cells may be using another receptor that may be recognizing other sequences as ligands in addition to the alpha5 beta1 integrin used to bind to the RGD sequence. This is currently being investigated using blocking antibodies to alpha 5 integrin in cell adhesion assays.

Recent studies by Chen and Culp (1996) and Xia and Culp (1994) have reported some cell adhesion to fragments EIIIB and EIIIA that were made as independent domains or in conjunction with their adjacent domains (7-B-8 and 11-A-12). However, the experimental conditions were very different with observation periods of 4 hours. The observation period in our experiments on cell adhesion was 1 hour for adhesion/spreading and only 15 minutes for the centrifugal assay. Our findings on the larger fragment 7-12+A differ from those reported by Xia and Culp (1994) where they report adhesion activity in domain EIIIA. The differences could be explained by the duration of adhesion assay (4 hours versus 1 hour in our study), concentration of the proteins used and cell types used. The cells that adhered to fragment 7$10+\mathrm{B}$ had spread just as well as pFN at equimolar concentrations. Immunostaining with antibodies to vinculin revealed a pattern of focal adhesion distribution in the cells plated on 7-10+B fragment that was comparable if not better to that seen on $\mathrm{pFN}$ at $50 \mathrm{nM}$ concentrations. Similarly, the distribution of the actin cytoskeletal network was comparable. However, the 7-10-B fragment 
showed slightly fewer focal adhesions and less well-organized actin network. The fact that the fragments 10-12 with and without EIIIA showed very little adhesion and virtually no spreading is not surprising given the previous findings that the RGD-containing domain alone is not sufficient to promote cell adhesion. Also, these findings suggest that domains 11 and 12 at the C-terminal end do not offer any kind of synergistic help to the putative RGDcontaining domain 10. Again, structural studies (Leahy et al., 1996; Main et al., 1992) imply the need for additional specific domains on the N-terminal end of the RGD loop, perhaps acting to stabilize the integrin. One of the interesting findings in the present study is the improved spreading and distribution of focal adhesions in cells plated on the larger 7-12 fragments. Here, the inclusion of EIIIB alone or both EIIIB and EIIIA appears to help adhesion and spreading of cells, while the inclusion of EIIIA in the absence of EIIIB keeps the cells from establishing good focal adhesions. The findings on the distribution of focal adhesions and actin stress fibers complement those of the centrifugal cell adhesion assay. This assay was designed to indirectly test the adhesive strength by examining the balance between centrifugal forces and cell substrate adhesion.. In non-adherent or weakly adherent substrata, cells may form a pellet due to the predominance of centrifugal force. With increasing strengths of cellsubstrate adhesion, cells resist centrifugal forces. Under the criteria used for scoring, a score of 3 means almost complete resistance to detachment by centrifugal forces in round-bottom wells. It should be emphasized that the cells were allowed to adhere only for 15 minutes. Cells on pFN showed scores of 3 at concentrations as low as $10 \mathrm{nM}$ and fragments 7-10+B and 710-B were next, showing scores of 3 at about $50 \mathrm{nM}$ concentrations. The larger fragments showed scores of 3 at $100 \mathrm{nM}$ concentrations only when EIIIB alone was included or both EIIIB and EIIIA were excluded. Cells on the fragment 7-12+A did not show scores of 3 even at the highest concentration tested $(600$ $\mathrm{nM})$. Similarly cells plated on the smaller fragments $10-12+\mathrm{A}$ and 10-12-A never reached scores of more than 2 even at 1,000 $\mathrm{nM}$ concentrations. Taken together, the data on distribution of focal adhesions and the centrifugal cell attachment assay suggest that the inclusion of EIIIB enhances the adhesion and spreading of cells while including EIIIA alone in the larger fragment negatively affects the adhesion and spreading of cells. It is tempting to speculate that the inclusion of both domains EIIIB and EIIIA in the FN molecule may be a biological mechanism of achieving cell adhesion to a degree that facilitates the process of locomotion where both adhesion and loss of adhesion are needed for the advancement of cells. The EIIIB+/EIIIA+ isoform of FN is common during embryonic development and wound healing (french-Constant and Hynes, 1989), both events where cell migration is important. More recently, it has been shown that the spliced domains EIIIB and EIIIA are included in the FN seen during the morphogenesis of 'FN dependent' structures such as somites, notochord and the vasculature (Peters and Hynes, 1996). Similarly, the expression of the EIIIA and EIIIB spliced variants in the adult tissues suggest that these isoforms of $\mathrm{FN}$ may be serving different functions (Peters et al., 1996).

This work was supported by National Institutes of Health Grant DE-07801 to IA. We thank Dr Harold Erickson for the plasma fibronectin, Drs Ken Yamada and Shin-Ichi Aota for providing us the antibodies to human FN, and Dr A. Belkin for the mAB to vinculin.

\section{REFERENCES}

Aota, S., Nomizu, M. and Yamada, K. M. (1994). The short amino acid sequence Pro-His-Ser-Arg-Asn in human fibronectin enhances cell adhesive function. J. Biol. Chem. 269, 24756-24761.

Aukhil, I., Joshi, P., Yan, Y. and Erickson, H. P. (1993). Cell- and heparinbinding domains of the hexabrachion arm identified by tenascin expression proteins. J. Biol. Chem. 268, 2542-2553.

Carnemolla, B., Leprini, A., Allemanni, G., Saginati, M. and Zardi, L. (1992). The inclusion of the type III repeat ED-B in the fibronectin molecule generates conformational modifications that unmask a cryptic sequence. $J$. Biol. Chem. 267, 24689-24692.

Chen, W. and Culp, L. A. (1996). Adhesion mediated by fibronectin's alternatively spliced $\mathrm{ED}_{\mathrm{b}}(\mathrm{EIIIB})$ and its neighboring type III repeats. Exp. Cell Res. 223, 9-19.

ffrench-Constant, C. and Hynes, R. O. (1989). Alternative splicing of fibronectin is temporally and spatially regulated in the chick embryo. Development 106, 375-388.

ffrench-Constant, C., Van de Water, L., Dvorak, H. and Hynes, R. O. (1989). Reappearence of an embryonic pattern of fibronectin splicing during healing in the adult rat. J. Cell Biol. 109, 903-914.

Humphries, M. J., Akiyama, S. K., Komoriya, A., Olden, K. and Yamada, K. M. (1987). Identification of two distinct regions of type-III connecting segment of human plasma fibronectin that promote cell type-specific adhesion. J. Biol. Chem. 262, 6886-6892.

Humphries, M. J., Akiyama, S. K., Komoriya, A., Olden, K. and Yamada, K. M. (1988). Neurite extension of chicken peripheral nervous system neurons on fibronectin: relative importance of specific adhesion sites in the central cell-binding domain and the alternatively spliced type III connecting segment. J. Cell Biol. 106, 1289-1297.

Hynes, R. O. (1990). Fibronectins. Springer-Verlag, New York.

Joshi, P., Chung, C. Y., Aukhil, I. and Erickson, H. P. (1993). Endothelial cells adhere to the RGD domain and the fibrinogen-like terminal knob of tenascin. $J$. Cell Sci. 106, 389-400.

Kawasaki, E. S. (1990) Amplification of RNA. In PCR Protocols -A Guide to Methods and Applications, 1st edn (ed. M. A. Innis et al.), pp. 21-27. Academic Press.

Kimizuka, F., Ohdate, Y., Kawase, Y., Shimijjo, T., Hashino, K., Goto, S., Hashi, H., Kato, I., Sekiguchi, K. and Titani, K. (1991). Role of type III homology repeats in cell adhesive function within the cell-binding domain of fibronectin. J. Biol. Chem. 266, 3045-3051.

Leahy, D., Aukhil, I. and Erickson, H. P. (1996). $2.0 \AA$ crystal structure of a four-domain segment of human fibronectin encompassing the RGD loop and synergy region. Cell 84, 155-164.

Main, A. L., Harvey, T. S., Baron, M., Boyd, J. and Campbell, I. D. (1992). The three-dimensional structure of the tenth type-III module of fibronectin: an insight into RGD-mediated interactions. Cell 71, 671-678.

Nagai, T., Yamakawa, N., Aota, S., Yamada, S., Akiyama, S., Olden, K. and Yamada, K. M. (1991). Monoclonal antibody characterization of two distinct sites required for function of the central cell-binding domain of fibronectin in cell adhesion, cell migration and matrix assembly. J. Cell Biol. 114, 1295-1305.

Peters, J. H. and Hynes, R. O. (1996). Fibronectin isoform distribution in the mouse. I - The alternatively spliced EIIIB,EIIIA and V segments show widespread codistribution in the developing mouse embryo. Cell Adhes. Commun. 4, 103-125.

Peters, J. H., Chen, B. and Hynes, R. O. (1996).Fibronectin isoform distribution in the mouse. II. Differential distribution of the alternatively spliced EIIIB, EIIIA and V segments in the adult mouse. Cell Adhes. Commun. 4, 127-148.

Sanger, F., Nicklen, S. and Coulson, A. R. (1977). DNA sequencing with chain-terminating inhibitors. Proc. Nat. Acad. Sci. USA 74, 5463-5467.

Schneider, G. and Burridge, K. (1994). Formation of focal adhesions by osteoblasts adhering to different substrata. Exp. Cell Res. 214, 264-269.

Studier, F. W., Rosenberg, A. H., Junn, J. J. and Dubendorff, J. W. (1990). Use of T7 RNA Polymerase to direct expression of cloned genes. Meth. Enzymol. 185, 60-89.

Xia, P. and Culp, L. A. (1994). Adhesion activity in fibronectin's alternatively spliced domain EDa (EIIIA) and its neighboring type-III repeats, Oncogenedependent regulation. Exp. Cell Res. 213, 253-265.

(Received 3 February 1997 - Accepted 10 July 1997) 